ELKOMIKA: Jurnal Teknik Energi Elektrik, Teknik Telekomunikasi, \& Teknik Elektronika

ISSN(p): 2338-8323 | ISSN(e): 2459-9638

DOI : http://dx.doi.org/10.26760/elkomika.v9i1.217

| Vol. 9 | No. 1 | Halaman 217 - 231

Januari 2021

\title{
Bi-Directional Data Communication using Visible Light Technology for Underwater Environment
}

\author{
LITA LIDYAWATI, ARSYAD RAMADHAN DARLIS, LUCIA JAMBOLA, \\ ADITYA ARIF PRIBADI
}

Teknik Elektro Institut Teknologi Nasional Bandung, Indonesia

Email: lita@itenas.ac.id

Received 23 November 2020 | Revised 13 Desember 2020 | Accepted 16 Desember 2020

\begin{abstract}
ABSTRAK
Pengembangan VLC (Visible Light Communication) dapat diimplementasikan ke dalam air. Pada penelitian ini dirancang sistem komunikasi menggunakan cahaya tampak untuk komunikasi dua arah di lingkungan bawah air. Sistem dirancang untuk mengirimkan sinyal digital dengan pengukuran jarak menggunakan filter warna. Terdapat dua link di bagian pemancar dan penerima. Untuk membedakan kedua link tersebut, data dari generator sinyal $3 \mathrm{kHz}$ akan diaplikasikan pada link 1 dan data dari pembangkit sinyal 4 kHz akan diaplikasikan pada link 2. Berdasarkan hasil pengujian, sistem menerima data secara optimal pada link 1

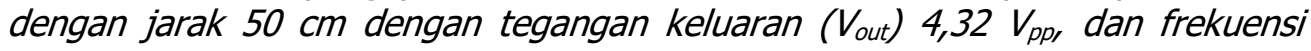
keluaran $\left(f_{o u t}\right) 3,121 \mathrm{kHz}$. Hasil pengukuran pada link 2 didapatkan tegangan keluaran sebesar 5,28 $\mathrm{V}_{p p}$ dan frekuensi keluaran $\left(f_{\text {out }}\right.$ ) sebesar 4,021 kHz. Pengukuran pada link 1 dengan filter warna biru didapatkan tegangan rata-rata sebesar 5,46 Vpp dan frekuensi keluaran $\left.\left(f_{\text {out }}\right)\right)$ sebesar 3,136 kHz. Sedangkan pengukuran link 2 dengan filter warna merah didapatkan tegangan rata-rata 5,11 $V_{p p}$ dan $f_{\text {out }} 4,054 \mathrm{kHz}$.
\end{abstract}

Kata kunci: color filter, bidirectional, data, UVLC

\begin{abstract}
The development of VLC (Visible Light Communication) can be implemented into underwater. In this research, we design a visible light communication system for two-way communication in the underwater environment. The system is designed to transmit digital signals with a distance measurement using a color filter. There are two links in transmitter and receiver part. To distinguish between those two links, data from the signal generator of $3 \mathrm{kHz}$ will be applied on link 1 and data from the the signal generator of $4 \mathrm{kHz}$ will be applied on link 2. The distance measurement system can receive the data optimally on link 1 with the distance of $50 \mathrm{~cm}$, an output voltage ( $V_{\text {out }}$ ) of $4.32 V_{p p}$, and output frequency (fout) of $3.121 \mathrm{kHz}$. The result measurement on link 2 obtains an output voltage of 5.28 Vpp and output frequency of $4.021 \mathrm{kHz}$. The measurement on link 1 which applied the blue color filter obtains the average voltage of $5.46 V_{p p}$ and the output frequency (fout) of $3.136 \mathrm{kHz}$, while on link 2 which applied the red color filter obtains an average voltage of $5.11 \mathrm{~V}_{p p}$ and $f_{\text {out }}$ of $4.054 \mathrm{kHz}$.
\end{abstract}

Keyword: color filter, bidirectional, data, UVLC 


\section{INTRODUCTION}

Visible Light Communication (VLC) is a data communication medium using visible light between $400 \mathrm{THz}(375 \mathrm{~nm})$ to $800 \mathrm{THz}(780 \mathrm{~nm})$. This communication technology utilizes light sources, namely Light Emitting Diode (LED) as transmitters, light as transmission media, and photodetectors as receivers. The applied LED in VLC consists of Optical Wireless Communication (OWC) which is connected using the visible light spectrum, where LEDs are applied with two functions, lighting and communication simultaneously. In VLC, communication is occurred by modulating the light intensity of the LED in such a way that it cannot be detected by the human eye, that it has no negative effect on the lighting function.

(Medina, 2015)

VLC research has demonstrated its ability to achieve very high data rates (close to $100 \mathrm{Mbps}$ according to the IEEE 802.15.7 standard up to a Gbps). The exponential growth in mobile data traffic effects the limitations of mobile communications using RF. Visible light spectrum covers hundreds of terahertz bandwidths that are completely unlicensed band for communication. VLC can substitute RF-based cellular communication systems when require high-capacity cellular data networks. Because it uses high frequencies, visible light cannot penetrate most objects and walls. This characteristic allows anyone to create tiny cells from the LED transmitter without the issue of interconnecting interference across walls and partitions. (Pathak, 2015)

LED is an electronic component that emits the monochromatic light when applied a forward voltage. LED is a family of diodes made of semiconductor materials. The colors of the light emitted by a LED depend on the type of semiconductor material it uses. LEDs can also emit infrared rays that are invisible as in TV Remote Controls of other devices. (Husaeni, 2018)

The visible light communication for underwater communication wherein narrowing the power-half angle of the light emitting diode (LED) which aims to increase the light intensity of the transmitter, and the use of a Single Photon Avalanche Diode (SPAD) on the receiver to improve detection sensitivity. LEDs have a variety of colors, including red, yellow, blue, white, green, orange and infrared. The color diversity of the LED depends on the wavelength and the semiconductors. Each LED's color requires a forward voltage (Forward Bias) to be able to turn it on. Photodiode is an electronic component that detects light. Photo Diodes will convert light into electric current. This electronic component is able to detect various types of light, ranging from infrared light, visible light, ultraviolet to X-rays. (Wang, 2016)

The bidirectional condition in optical communication occurs while each link using a wavelength which is represented by a color LED and a color filter. On the proposed system, water tank is utilized to represent the underwater environment. The first link, the VLC transmitter with the single color LED transmits an information signal through the water tank that represents an underwater environment and then is received by VLC receiver via a color filter. The same condition occurs for the second link where the information signal is transmitted from VLC transmitter toward VLC receiver using the same or different color LED. This research is prospective to give the contribution to find the best of the color pair for the bidirectional VLC system in underwater environment. This system can support the bidirectional communication between vehicles, divers, and devices in the underwater environment. (Darlis, 2018)

Visible light communication (VLC) has the potential to serve as a high-speed underwater wireless connectivity solution to support real-time image and video transmission. Underwater propagation medium imposes additional challenges on the design of VLC systems which were 
Bi-Directional Data Communication using Visible Light Communication technology for Underwater Environment

originally proposed for indoor applications. Temperature and salinity fluctuations result in fluctuations of the refractive index of seawater and eventually introduce turbulence-induced fading. In this paper, to mitigate the effects of fading, we propose transmit laser selection for a diver-to-diver underwater VLC link where the transmitter has multiple laser sources and the receiver has one photodetector. The source with the highest received instantaneous signal-to-noise ratio is selected for transmission while the remaining sources remain idle. We derive a closed-form expression for asymptotical bit error rate over log-normal distributed underwater turbulence channels and derive the achievable diversity order. We further present simulation results to confirm our derivations and diversity gain analysis. (Ellamasie, 2019)

Power domain non-orthogonal multiple access (NOMA) is introduced in underwater visible light communication (UVLC) to improve the spectrum efficiency and the sum transmission rate. A NOMA-UVLC system employing two LEDs with different colors is designed to improve the propagation efficiency, by bearing different information on different carriers. We experimentally investigate the BER performance of the system in air and water environment, respectively. The results demonstrate that the proposed NOMA system works better in underwater environment than in air environment. The total transmission rate in underwater environment achieves $117.4 \mathrm{Mb} / \mathrm{s}$ when employing red/blue LEDs in the proposed system, and $93.9 \mathrm{Mb} / \mathrm{s}$ when employing red/green LEDs in the proposed system, with a transmission distance of $1 \mathrm{~m}$. (Chen, 2020)

Since the two branches of the DBMLP can compensate linear and nonlinear distortion in the received signal, respectively, the complexity of the neural network is reduced and the performance of the post-equalizer is improved. Compared with multi-layer perceptron (MLP) based PE the space complexity of DBMLP based PE reduces $59.5 \%$, and the bit error rate (BER) at $3.1 \mathrm{Gbps}$ reduces $45.5 \%$. The reduction of spatial complexity helps the application of post-equalizer based on neural network algorithms in practical communication systems. Meanwhile, increased BER performance helps underwater visible light communication (UVLC) systems reach higher rates. We achieved $3.2 \mathrm{Gbps}$ in the 64 quadrature amplitude modulation (64QAM)-carrierless amplitude and phased modulation (CAP) modulated UVLC system using DBMLP based PE. This is the highest record of data rate in the field of one single chip blue LED based UVLC system. (Zhao, 2020)

An underwater visible light communication system utilizing QAM-DMT and Multi-PIN reception to do the MRC receiving. The transmitter is a $600 \mu \mathrm{m} \times 600 \mu \mathrm{m}$ green-emitting silicon substrate LED which has a peak emission wavelength of $521 \mathrm{~nm}$. We study the effect of quiescent working point of the LED, the zero-padding number, the ISFA tap number and the ratio of two receivers on the BER performance of our VLC system. We notice that the ratio of two receivers can remarkably affect the performance of our system. They also measured the BER performance versus the bandwidth of the signal in 32QAM, 64QAM and 128QAM modulation format and find the highest data rate of the system can reach 2.175 $\mathrm{Gb} / \mathrm{s}$. It is the highest data rate in under pure water VLC system using commercial LED and a PIN receiver. (Wang, 2018)

The propagation of optical signal under water is affected by three major degrading phenomena, namely absorption, scattering, and turbulence. The statistical distribution of turbulence-induced fading though has not yet been well characterized. In this paper, in order to investigate the effect of different probability density functions (PDFs) proposed for the statistical behavior of fading under water, we evaluate the performance of underwater wireless optical communication (UWOC) systems with respect to the well-known statistical distributions for the optical turbulence such as lognormal, Gamma, K, Weibull, and 
exponentiated Weibull distributions. For the sake of accuracy, they adopt a general channel model where both absorption and scattering effects are appropriately taken into account based on the Monte Carlo (MC) numerical method, and consider the fading effect as a multiplicative coefficient with the aforementioned PDFs. They derive the closed-form expressions for the average bit error rate (BER) and outage probability, as the system performance metrics, with respect to all of the channel degrading effects and the aforementioned statistical distributions. The results demonstrate that as the turbulence strength, measured by the scintillation index value, increases, the gap between the system performance predicted by different statistical distributions becomes more, and this gap mainly appears in the form of changing the slope of average BER or outage probability curves versus the average transmitted power per bit. This further emphasizes the importance of accurate channel models for the design of UWOC systems. (Syarifzadeh, 2018)

The performance of underwater wireless optical multiple-input multiple-output communication system combining spatial modulation (SM-UOMIMO) with flag dual amplitude pulse position modulation (FDAPPM). Channel impulse response for coastal and harbor ocean water links are obtained by Monte Carlo (MC) simulation. Moreover, they obtain the closedform and upper bound average bit error rate (BER) expressions for receiver diversity including optical combining, equal gain combining and selected combining. And a novel adaptive power allocation algorithm (PAA) is proposed to minimize the average BER of SMUOMIMO system. Our numeric results indicate an excellent match between the analytical results and numerical simulations, which confirms the accuracy of our derived expressions. Furthermore, the results show that adaptive PAA outperforms conventional fixed factor PAA and equal PAA obviously. Multiple-input single-output system with adaptive PAA obtains even better BER performance than MIMO one, at the same time reducing receiver complexity effectively. (Huang, 2018)

The next reference paper was about the performance limits of underwater visible light communication (UVLC) systems. Here, they developed a closed-form path loss expression as a function of transceiver parameters and water type. Thus, utilize this new expression to determine the maximum achievable link distance for UVLC systems in pure sea, clear ocean, coastal water, and harbor water. The results demonstrate that the maximum achievable distance is limited to a few tens of meters. This necessitates the deployment of relayassisted UVLC systems to extend the transmission range. They consider both detect-andforward and amplify-and-forward relaying. For each relaying method, we first consider a dual-hop UVLC system and determine optimal relay placement to minimize the bit error rate (BER). Then, they consider a multi-hop system with equidistant relays and determine the maximum achievable distance for a given number of hops to satisfy a targeted end-to-end BER. (Elamassie, 2018)

This paper was discussed about the performance of vertical underwater visible light communication (VLC) links. In vertical links, water properties such as temperature and salinity change with the depth. This leads to changes in the refractive index eventually affecting the variance of turbulence-induced fading. Considering such variations with respect to depth, we first model the vertical underwater link as the concatenation of multiple layers and develop a statistical turbulence channel model based on the cascaded structure of fading coefficients. Then, we use this expression to formulate a closed form expression for bit error rate performance. We confirm the analytical derivations through simulations. (Elamassie, 2018) 
In this study we propose a bi-directional communication using the principle of visible light communication with LED lights, where the system can transmit data in digital signals under the water environment.

\section{METHODS}

LEDs have been widely deployed for illumination, due to their high performance and energy efficiency properties compared to the conventional descent and fluorescent lamps. (Komine, 2014). In addition, many advantages offered by applying LED such as high frequency response, free spectrum license and high security, whereby people can access the internet through the same visible light.

The system is designed uses 4 pieces of hardwares, which are 2 transmitters and 2 receivers, each hardware is measured to obtain the desired performance. Afterwards, the transmitter and receiver are combined in order to gain a desired signal. Furthermore, apply the LED light on the transmitter circuit and photodiode on the receiver circuit. The diagram of the primary system is shown in Figure 1.

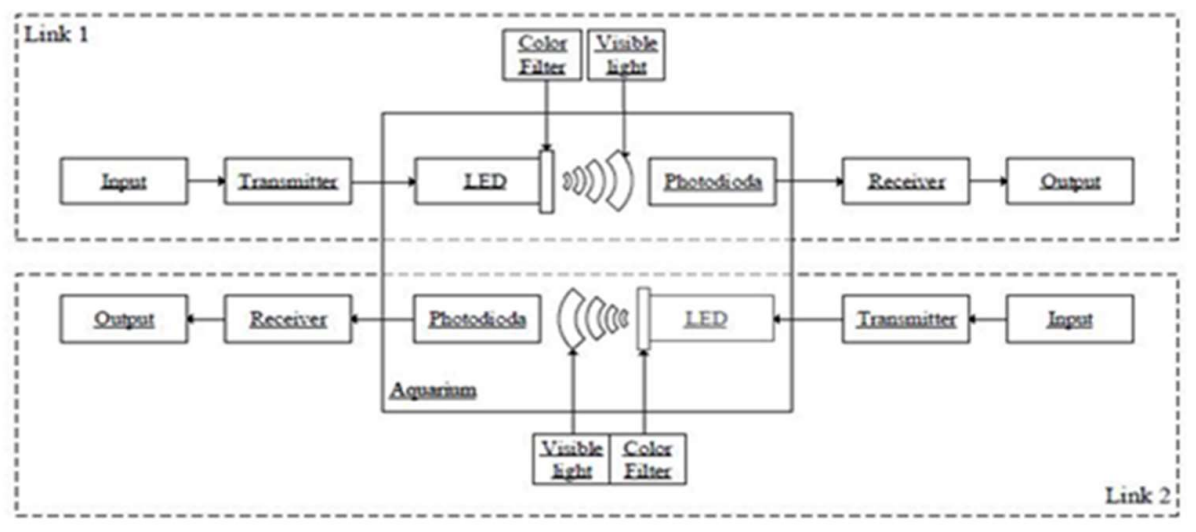

Figure 1. Diagram Block System

In the transmitter section, the input will send the data that will be processed through a series of transmitters. Afterwards, it will be transmitted through visible light using the LEDs component. The LEDs function to convert electrical energy into light. In the receiver section, the light which contains information must be captured by a component that is able to convert light into an electrical signal.

Visible light communication is free space optical communication and line of sight (LOS) is the common link between two points in optical wireless communication system, where the transmitter directs the visible light beam in a straight and unobstructed path to the receiver

(He, 2013). In this technology, LEDs are used as a transmitter, the water as a transmission medium and the photodiodes as a receiver.

\subsection{Implementation of VLC Transmitter}

VLC is typically implemented using white LED light bulbs at the transmitter. These devices are normally used for illumination only by applying a constant current. On the transmitter's side, the information signal or data that is transmitted into a form of light will be generated by the LED and passed to the color filter to avoid the wavelength interference. 
The signal generated by the LED is passed to a color filter which aims to determine the wavelength of the visible light emitted. In this transmitter section consists of several components i.e. audio generator, LED, transmitter with a simple RC circuit. The transmitter is connected to generator signal that will send an information signal. The transmitter circuit can be seen at Figure 2.

Table 1. Transmitter Characteristic

\begin{tabular}{|c|c|c|}
\hline No & Description & Value (unit) \\
\hline 1 & Power Supply & 5 (volts) \\
\hline 2 & LED & 5 (volts) \\
\hline 3 & Resistor & 100 ohm \\
\hline
\end{tabular}

The LED in the sending circuit functions as a transmitter of an information signal which is converted into visible light which will then be transmitted through the water and received by the photodiode in the receiver circuit.

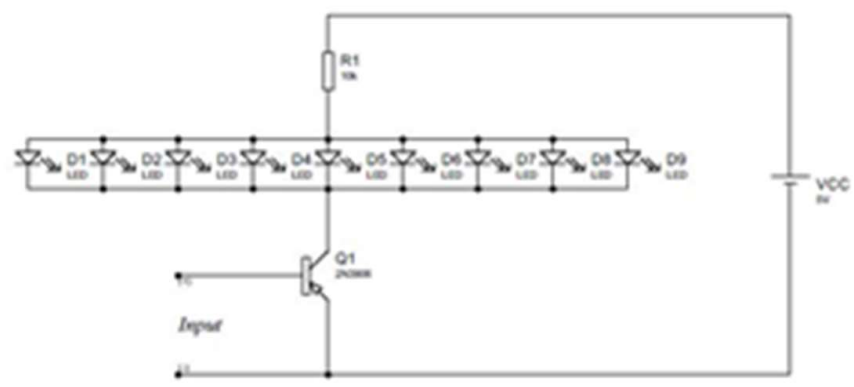

Figure 2. Transmitter Circuit

\subsection{Implementation of VLC Receiver}

The photodiode must be placed on the front of the receiver in order to receive the desired signal from the transmitter. This is the purpose of the photodiodes as they react to the light emitted from the LEDs and allow for current to flow to the rest of the circuit receiver. When there is no light emitted from LEDs the photodiodes do not allow current to flow through to the receiver. Figure 3 shows the receiver circuit that contains photodiode and resistors.

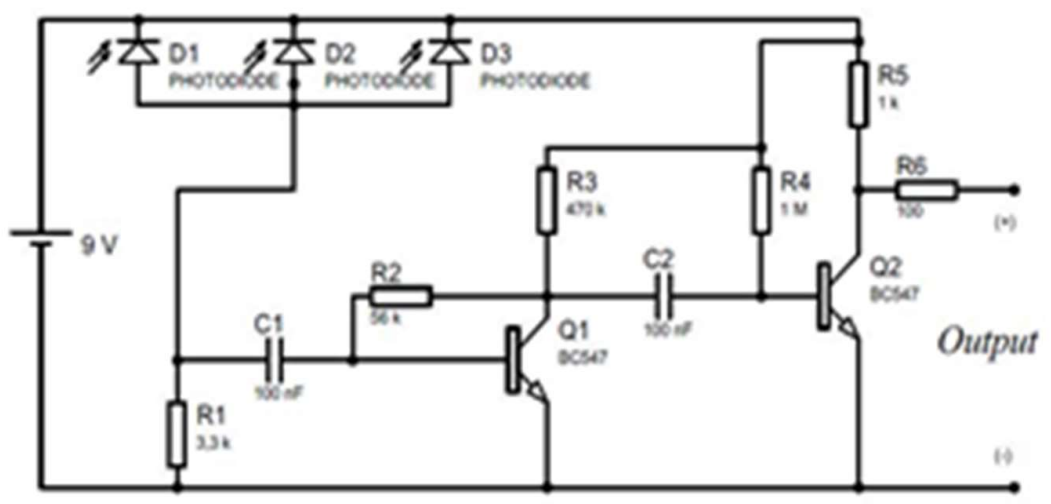

Figure 3. Receiver Circuit 
Bi-Directional Data Communication using Visible Light Communication technology for Underwater Environment

Table 2. Receiver Characteristic

\begin{tabular}{|c|l|l|}
\hline No & \multicolumn{1}{|c|}{ Description } & \multicolumn{1}{c|}{ Value (unit) } \\
\hline 1 & Power Supply & 9 (volts) \\
\hline 2 & Photodiode & $5 \mathrm{~mm}$ \\
\hline 3 & Resistor & $100 \mathrm{ohm}-470 \mathrm{~K}$ \\
\hline 4 & Capacitor & $100 \mathrm{nF}$ \\
\hline
\end{tabular}

\section{RESULTS AND DISCUSSION}

The VLC system is different from the other communication systems and it will be summarized these differences in the following tables.

\section{Table 3. Comparison Between VLC and RF Communication}

\begin{tabular}{|c|l|l|}
\hline Property & \multicolumn{1}{|c|}{ VLC } & \multicolumn{1}{c|}{ RF } \\
\hline Visibility & Yes & No \\
\hline Frequency & $\begin{array}{l}400 \quad \mathrm{THz}- \\
790 \mathrm{THz}\end{array}$ & $850 \mathrm{~nm}-950 \mathrm{~nm}$ \\
\hline Power & Relative low & Medium \\
\hline $\begin{array}{c}\text { Coverage } \\
\text { distance }\end{array}$ & Short & Medium \\
\hline
\end{tabular}

In order to obtain the best performance, we conducted the experiment to measure the maximum distance where the receiver is not able to receive the signal. The measurement was made on the voltage at several distances in order to know at what distance the receiver (photodiode) will not receive any data from the transmitter (LEDs). There are two measurement methods i.e. measurement without color filter and the measurement with color filter. Figure 4 shows the measurement method of the system in the air without color filter.

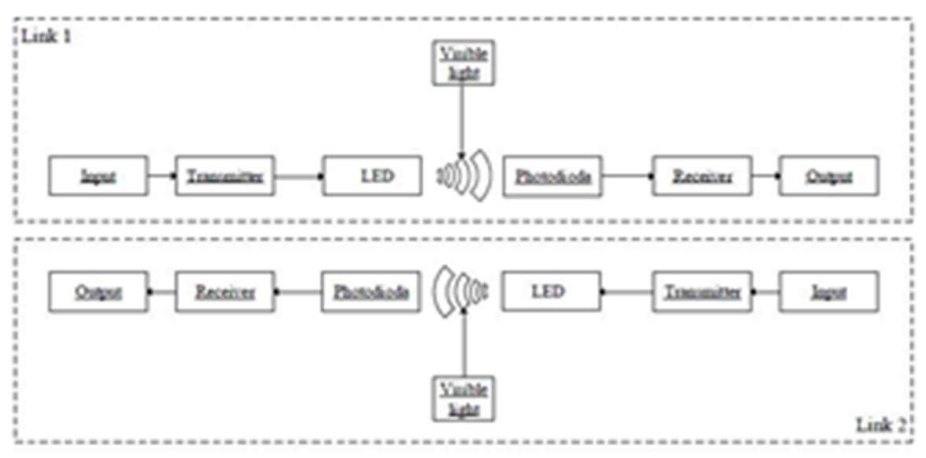

Figure 4. The Measurement Method of the system in the Air

This measurement aims to determine the reliability of the system in sending data in the air. The digital signal is generated by a signal generator with a frequency of $3 \mathrm{kHz}$ and amplitude of $5 \mathrm{Vpp}$, while on link 2 the frequency is $4 \mathrm{kHz}$ and the amplitude is $5 \mathrm{Vpp}$. Figure 5 shows the result of the measurement of the system against the distance bi-directional in the air, the system can receive the signal at a distance of $120 \mathrm{~cm}$ in the air. However, the captured signal is in poor quality. 


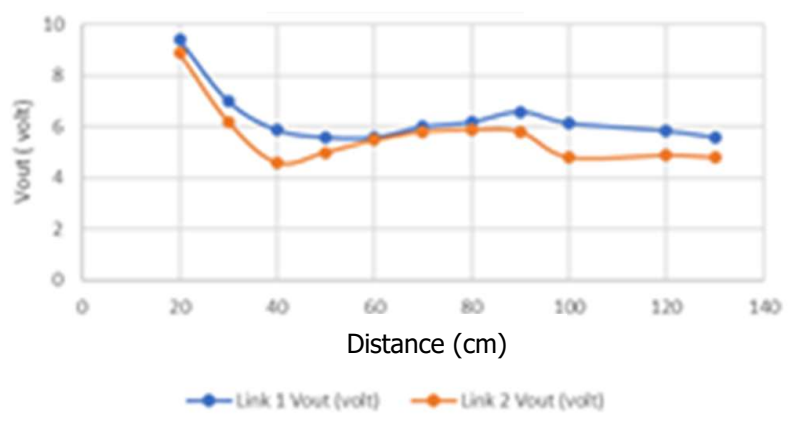

Figure 5. Graph of Distance In Response to Voltage in the Air

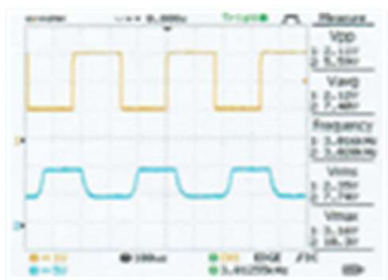

(a)

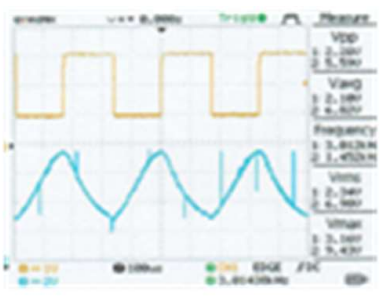

(b)

Figure 6. The Signal test at Link 1 (a) $50 \mathrm{~cm}$ (b) $120 \mathrm{~cm}$

Figure 7 shows the result of the measurement of the system at link 2 against the distance bidirectional in the air, the system can receive a signal at a distance of $120 \mathrm{~cm}$ in the air however the quality of the signal that is captured is of poor quality, the voltage value below 5 Vp-p.

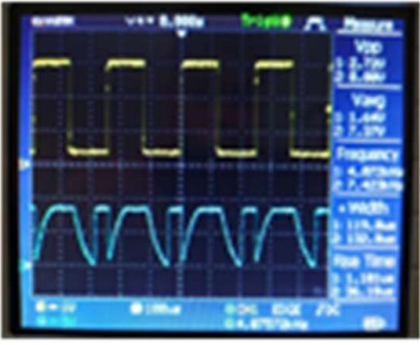

(a)

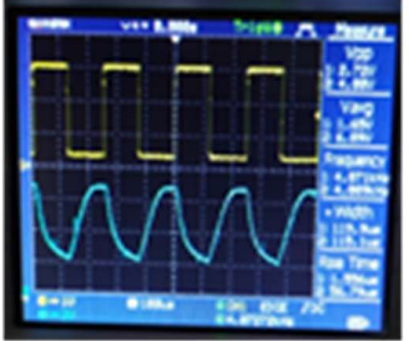

(b)

Figure 7. The Signal Test at Link 2 (a) $50 \mathrm{~cm}$ (b) $120 \mathrm{~cm}$

Furthermore, the measurement method is applied under the water environment. The data is generated from the signal generator by differentiating the frequency of $3 \mathrm{kHz}$ and the input link 1 has an amplitude of $5 \mathrm{Vp}$-p. Moreover, the data of $4 \mathrm{kHz}$ and an amplitude of $5 \mathrm{Vp}-\mathrm{p}$ on link 2, with the distance between the LED and the photodiode is being changed. Figure 8 shows the measurement method for the underwater environment. 
Bi-Directional Data Communication using Visible Light Communication technology for Underwater Environment

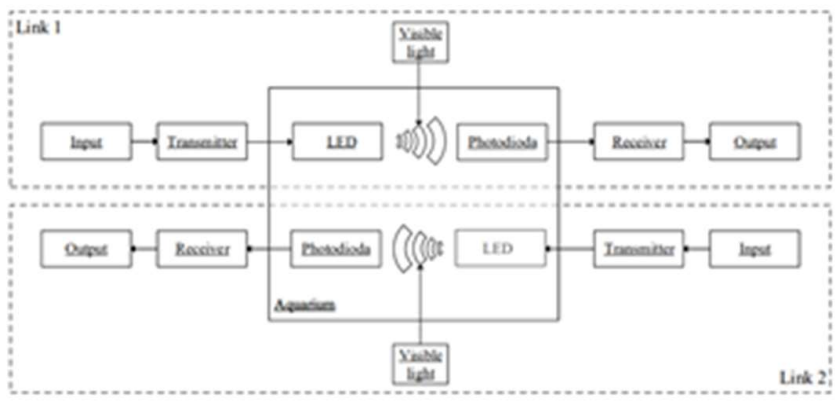

Figure 8. The Measurement Method of the System under the Water Environment

The system gains the optimal distance thus the transmitted signal can be received properly by the receiver. It is shown that at a distance of $50 \mathrm{~cm}$ the data is received properly by the receiver at link 1 and link 2. The output voltage (Vout) is $4.32 \mathrm{Vpp}$ and the output frequency (Fout) is $3.121 \mathrm{kHz}$ on link 1 . The signal on link 2 obtains the output voltage (Vout) of 5.28 Vpp and the output frequency (Fout) of $4.021 \mathrm{kHz}$. Figure 9 shows the signal measurement with different distances.

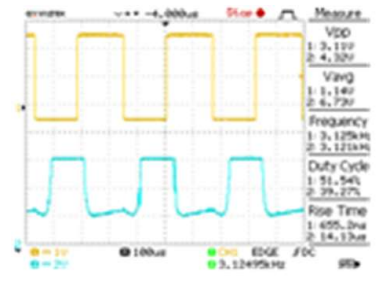

(a)

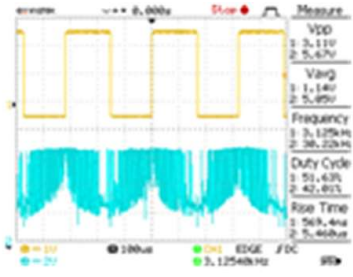

(b)

Figure 9. The Measurement Signal between Transmitter and Receiver at Link 1

(a) $50 \mathrm{~cm}$ (b) $100 \mathrm{~cm}$

The receiver circuit is located at the distance of $50 \mathrm{~cm}$. According to the figure 9 and 10, the signal quality can be received properly even almost resembling the sender's signal while the $100 \mathrm{~cm}$ pad photodiode can still catch the signal but the signal quality is not good in conjunction with the decreasing of signal level below $1 \mathrm{Vpp}$. It is due to transmissions with digital signals can only be sent over a narrow distance. Hereafter, the signal received at link 2 is shown in figure 10.

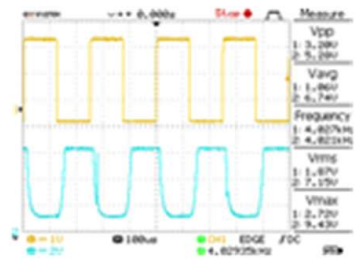

(a)

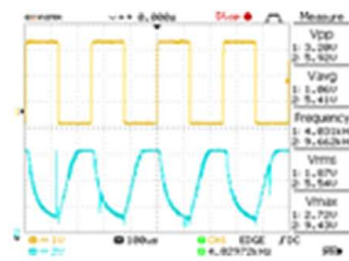

(b)

Figure. 10 The Measurement Signal between Transmitter and Receiver at Link 2 (a) 50 cm (b) $100 \mathrm{~cm}$ 


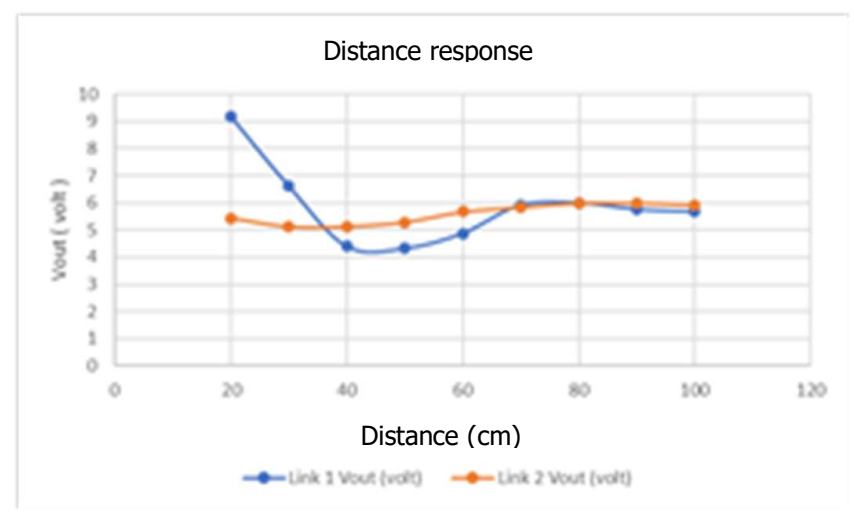

Figure 11. The Graph of Signals with Difference Distance

Furthermore, the final measurement method is applied to the color filter on the LEDs. In this measurement, to find out the value of each filter link used, applied 3 color filters which consist of a red filter, a blue filter and a green filter. The color filter is located on the transmitter, each color is tested at a distance of $20 \mathrm{~cm}-100 \mathrm{~cm}$ with a signal amplitude of 5 Vp-p. Link 1 uses a frequency of $3 \mathrm{kHz}$ and link 2 uses a frequency of $4 \mathrm{kHz}$ with the distance between the LED and the photodiode varying. The purpose of this measurement is to analyze the system which do not apply the color filter and to find the best link to perform the information exchange simultaneously.

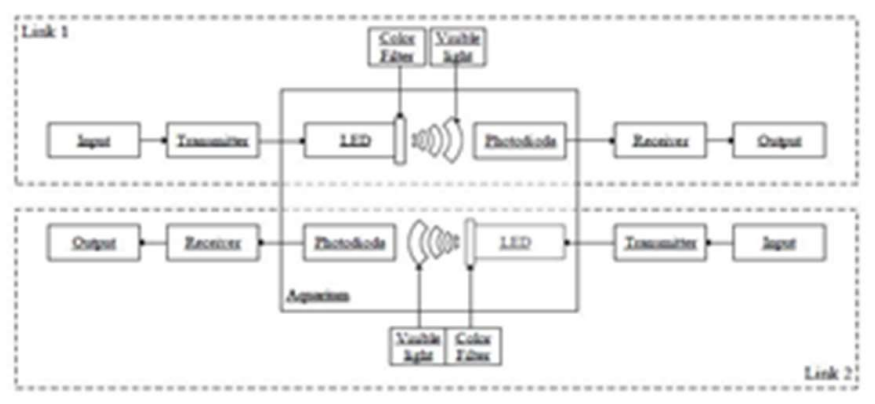

Figure 12. The Measurement method with Color Filter

Figure 12 is the block diagram of the color filter utilization system in measurements using color filters. Figure 13 to 15 are the measurement results of the red filter on link 1 with a variety of red, green and blue filters on link 2 .

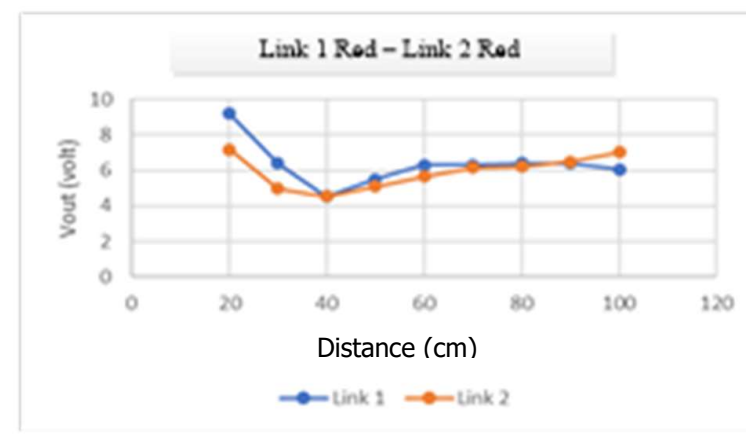

Figure 13. The Graph of Distance Response to voltage using Red - Red Color Filter 
Bi-Directional Data Communication using Visible Light Communication technology for Underwater Environment

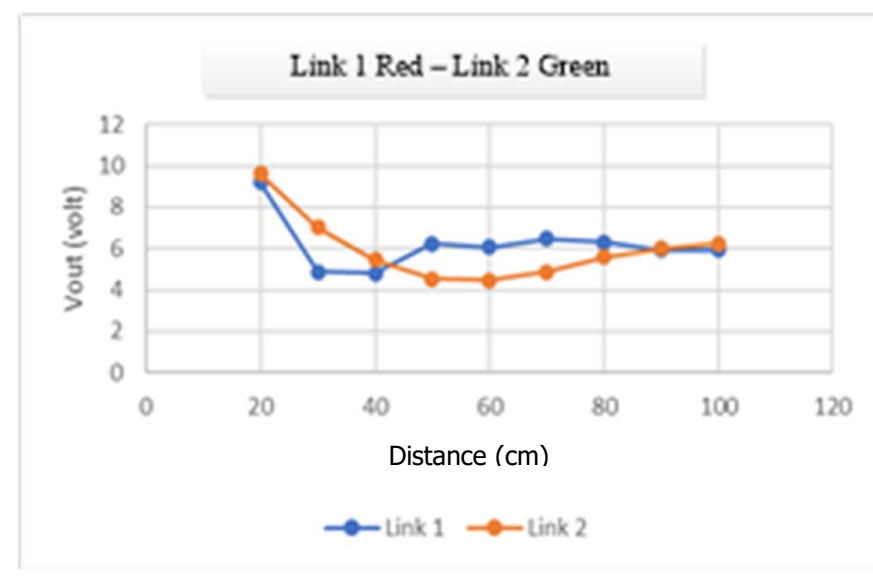

Figure 14. The Graph of Distance Response to voltage using Red - Green Color Filter

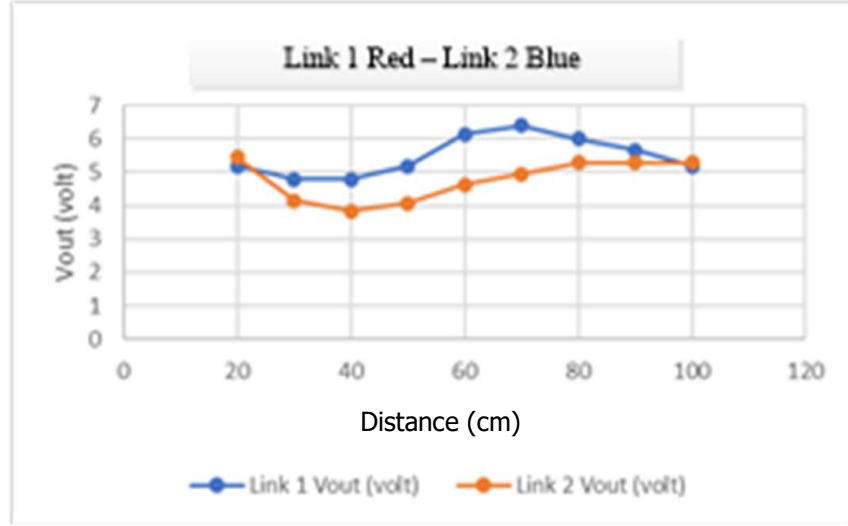

Figure 15. The Graph of Distance Response to voltage using Red - Blue Color Filter

There are 3 graphs for the distance response from each link, the blue line is the response to link 1 and the orange line is the response to link 2 . The red - red color filter indicates the first color mentioned is link 1 where there is a color filter on the transmitter part, as well as the second color is link 2 where there is a color filter found on the transmitter part. In this measurement, the filter that is modified is the filter on link 2.

From the graphs show the optimal distance obtained at a distance of $50 \mathrm{~cm}$ at each $\neg$ link, because at that distance the received signal almost resembles the signal at the sender. As shown in this test when a distance of $50 \mathrm{~cm}$ is obtained using a red-red filter, i.e. Vout 5.51 Vp-p and Fout $3.131 \mathrm{kHz}$ on link 1, and on link 2 Vout $5.11 \mathrm{Vp}-\mathrm{p}$ and Fout 3,990 kHz. Then the red - green filter, link 1 on the red filter gets Vout $6.23 \mathrm{Vp}-\mathrm{p}$ and Fout 3,286 kHz, link 2 on the green filter Vout $4.55 \mathrm{Vpp}$ and Fout $4020 \mathrm{kHz}$. The red - blue filter test results obtained on link 1 on the red filter, namely Vout $5.19 \mathrm{Vp}-\mathrm{p}$ and Fout $3.181 \mathrm{kHz}$, link 2 on the blue filter obtained Vout 4.07 Vp-p and Fout $4.012 \mathrm{kHz}$.

Furthermore, the results of measurements using a green filter will conduct on link 1 and a variety of green, red and blue filters on link 2. Figure 15 to 17 are the measurement results of the Green Filter on link 1 with a variety of green, red and blue filters on link 2. 


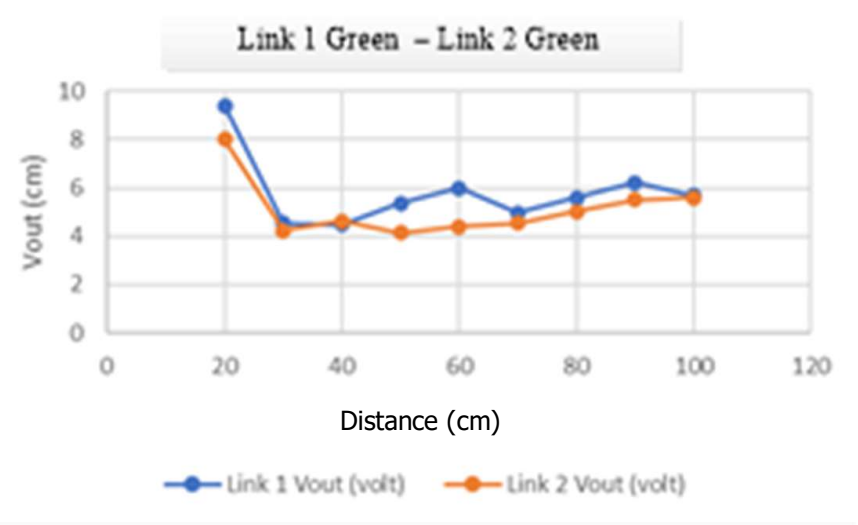

Figure 16. The Graph of Distance Response to voltage using Green - Green Color Filter

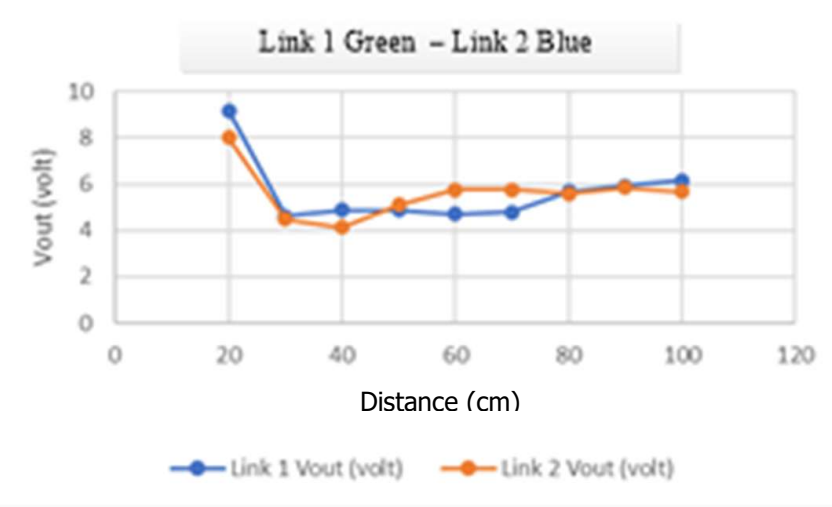

Figure 17. The Graph of Distance Response to voltage using Green - Red Color Filter

The blue line indicates the response from link 1 and the orange line indicates the response graph on link 2. The explanation of the writing of the title on each graph where green - red indicates the first color mentioned is link 1 where there is a color filter on the transmitter part, as well as the second color is link 2 where there is a color filter found on the transmitter part. In this measurement, the filter that is modified is the filter on link 2.

The measurement results show that the optimal distance is obtained at a distance of $50 \mathrm{~cm}$ on each link. When the distance is $50 \mathrm{~cm}$, the results are obtained using a green-green filter, namely, Vout $5.36 \mathrm{Vp}-\mathrm{p}$ and Fout $3.074 \mathrm{kHz}$ on link 1, and on link 2 Vout $4.1 \mathrm{Vp}-\mathrm{p}$ and Fout $4.020 \mathrm{kHz}$. Thus, the green - red filter, link 1 on the green filter gets Vout $5.11 \mathrm{Vp}-\mathrm{p}$ and Fout $3.079 \mathrm{kHz}$, link 2 on the red filter Vout $4.1 \mathrm{Vp}-\mathrm{p}$ and Fout $4.020 \mathrm{kHz}$. The green - blue filter test results obtained on link 1, the green filter of Vout $4.88 \mathrm{Vp}-\mathrm{p}$ and Fout $3.030 \mathrm{kHz}$, link 2 on the blue filter obtained Vout $4.07 \mathrm{Vp}-\mathrm{p}$ and Fout $4.012 \mathrm{kHz}$.

The final method is the measurement of a blue filter on link 1 and a variety of hunting color filters, red and green on link 2. Figure 18 to 20 show the graph of the measurement results of the red filter on link 1 with a variety of red, green and blue filters on link 2 . 
Bi-Directional Data Communication using Visible Light Communication technology for Underwater Environment

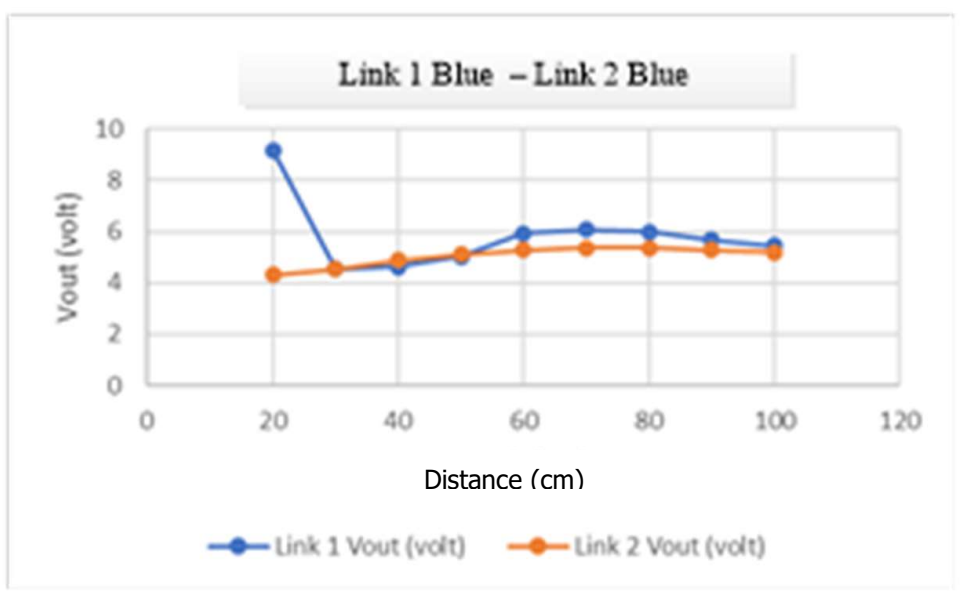

Figure 18. The Graph of Distance Response to voltage using Blue - Blue Color Filter

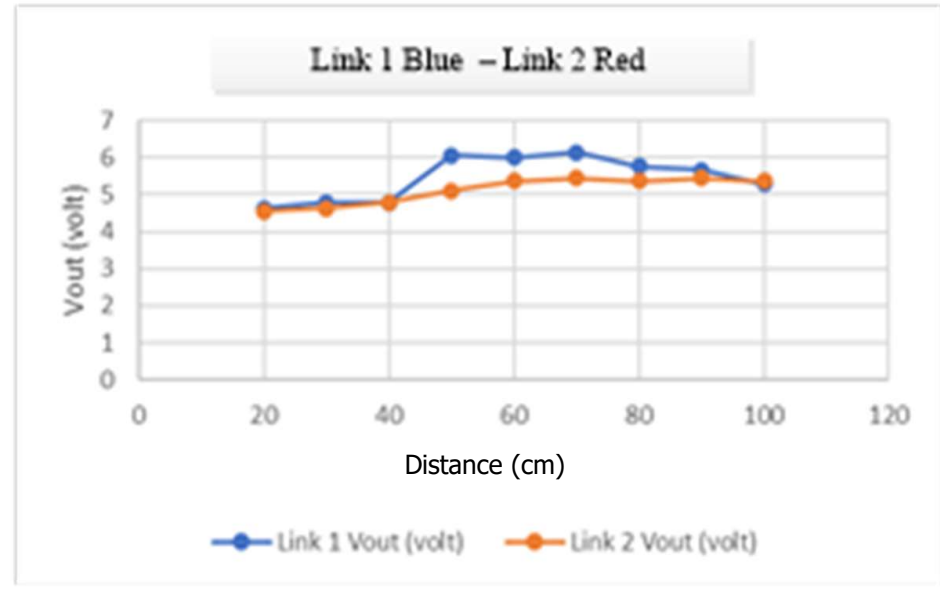

Figure 19. The Graph of Distance Response to voltage using Blue - Red Color Filter

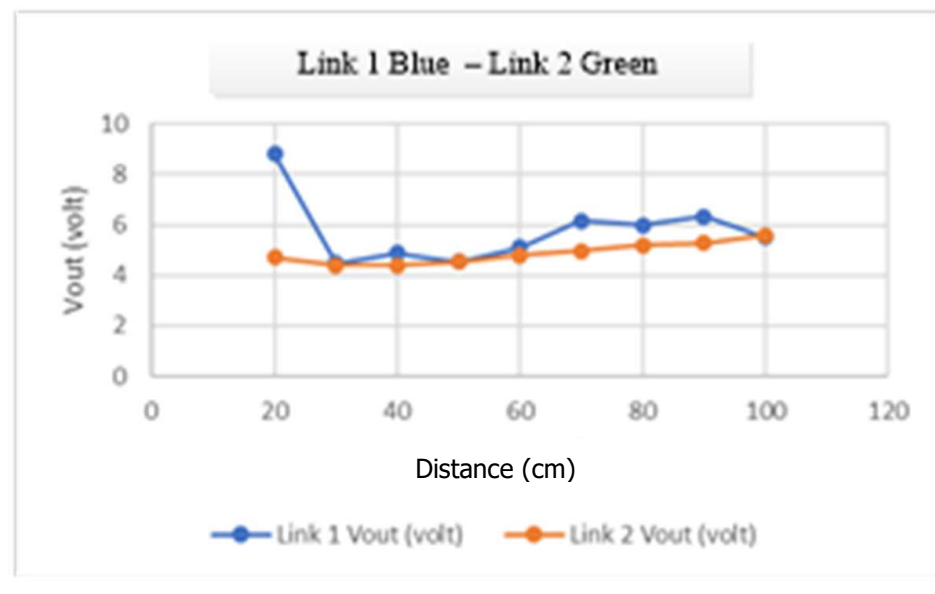

Figure 20. The Graph of Distance Response to voltage using Blue - Green Color Filter

The measurement results show that the optimal distance is obtained at a distance of $50 \mathrm{~cm}$ at each link. When the distance is $50 \mathrm{~cm}$, the results obtained using a blue - blue filter, 
namely, Vout $5.33 \mathrm{Vp}-\mathrm{p}$ and Fout $3.003 \mathrm{kHz}$ on link 1, on link 2 Vout $5.11 \mathrm{Vp}-\mathrm{p}$ and Fout $4.006 \mathrm{kHz}$. Thus, the blue - red filter, link 1 on the blue filter gets Vout $6.07 \mathrm{Vp}-\mathrm{p}$ and Fout $3.027 \mathrm{kHz}$, link 2 on the red filter Vout $5.11 \mathrm{Vp}-\mathrm{p}$ and Fout $4.032 \mathrm{kHz}$. The blue - green filter test results obtained on link 1 on the blue filter, namely Vout $4.55 \mathrm{Vp}-\mathrm{p}$ and Fout $3.071 \mathrm{kHz}$, link 2 on the blue filter obtained Vout $5.11 \mathrm{Vp}-\mathrm{p}$, and Fout $4.005 \mathrm{kHz}$.

Based on the measurement results of the nine color pairs tested, from the data taken the color pairs that can be used as link 1 and link 2 in bi-directional communication are pairs with blue - red filters where the blue filter on link 1 Vout is $5.46 \mathrm{Vp}-\mathrm{p}$ Fout 3,136 kHz and link 2 with a red filter Vout $5.11 \mathrm{Vp}$-p Fout 4,054 kHz. This could be because the blue filter on link 1 has good characteristics in communication in the underwater environment and the color on the color filter on link 2 has a good sensitivity value on the photodiode used.

\section{CONCLUSION}

At the end of this research we were able to send and receive the data properly. Measurements with a bi-directional color filter in the underwater environment, obtained a color pair between links that has a good response that is the blue filter pair on link 1 .

\section{ACKNOWLEDGMENT}

This work was financially supported by the Ministry of Research, Technology \& Higher Education of Republic Indonesia under research grant number B/87/E3/RA.00/2020, through the PUPT 2020 scheme.

\section{DAFTAR RUJUKAN}

Chen, D., Wang, Y., Jin, J., Lu, H., Wang, J. (2020) An experimental study of NOMA in underwater visible light communication system. Optics Communications, 475.

Darlis, A. R., Widura, A., Andrian, M.R. (2018). Bidirectional Underwater Visible Light Communication. International Journal of Electrical and Computer Engineering (IJECE), 8(6), 5203-5214.

Elamassie, M., Al-Nahhal, M., Kizilirmak, R.C., Uysal, M. (2019). Transmit Laser Selection for Underwater Visible Light Communication Systems. 2019 IEEE $30^{\text {th }}$ Annual International Symposium on Personal, Indoor and Mobile Radio Communication (PIMRC). DOI: 10.1109/PIMRC.2019.8904100.

Elamassie, M., Miramirkhani, F., Uysal, M. (2018). Performance Characterization of underwater visible light communication. IEEE Transactions on Communications, $671), 543-552$.

Elamassie, M., Uysal, M. (2018). Performance Characterization of vertical underwater VLC links in the presence of turbulence. 11th International Symposium on Communication 
Bi-Directional Data Communication using Visible Light Communication technology for Underwater Environment

Systems, Networks\&Digital Signal Processing (CSNDSP). DOI: 10.1109/CSNDSP.2018.8471888. September 27th 2018. Publisher: IEEE

He, Y. J., Ding. L., Gory, Y., Wang, Y. (2013). Real-time Audio \& Video Transmission Based on Visible Light Communication. Optics and Photonics Journal, 153-157.

Huang, A., Tao, L., Niu, Y. (2018). Underwater wireless optical MIMO system with spatial modulation and adaptive power allocation. Optics Communications, 412.

Husaeni, D. F., Darlis, A. R., Lidyawati, L., \& Nataliana, D. (2016). Implementasi Visible LIght Communication (VLC) pada Sistem Komunikasi. Elkomika, 1(1), 13 - 25.

Komine, T., Nakagawa, M. (2014). Fundamental Analaysis for Visible Light Communication using LED Lights. IEEE Transaction on Consumer Electronics, 100-107.

Medina, C., Zambrano, M, Navarro, K. (2015). LED Based Visible Light Communication Technology, Applications and Chanllenges a Survey. IJAET, DOI: 10.7323/ijaet/V8_iss4.

Pathak, P., Feng, X., Hu, P. Mohapatra. (2015). Visible Light Communication, Networking, and Sensing: A Survey, Potential and Challenges in IEEE Communications Surveys \& Tutorials, 174), 2047-2077.

Sharifzadeh, M., Ahmadirad, M. (2018). Performance Analysis of underwater wireless optical communication systems over a wide range of optical turbulence. Optics Communications, 427.

Wang, C., Yu, H. Y., Zhu, Y. J. (2016). Underwater Visible Light Communication a Long Distance Underwater Light Communication System with Single Photon Avalanche Diode. IEEE Photonics Journal, \&(5), 1-11. DOI: 10.1109/JPHUT.2016.2602330.

Wang, F., Liu Y., Jiang, F., Chi, N. (2018). High speed underwater visible light communication system based on LED employing maximum ratio combination with multi-PIN reception. Optics Communications, 425. .

Zhao, Y., Zou, P., Chi, N. (2020). 3.2 Gbps underwater visible light communication system utilizing dual-branch multi-layer perceptron based post-equalizer. Optics Coomunication, 460. 